NEWS

\title{
Publish or perish in China
}

The pressure to rack up publications in high-impact journals could encourage misconduct, some say.

The latest in a string of high-profile academic fraud cases in China underscores the problems of an academic-evaluation system that places disproportionate emphasis on publications, critics say. Editors at the UK-based journal Acta Crystallographica Section E last month retracted 70 published crystal structures that they allege are fabrications by researchers at Jinggangshan University in Jiangxi province. Further retractions, the editors say, are likely.

Chinese universities often award cash prizes, housing benefits or other perks on the basis of high-profile publications, and the pressure to publish seems to be growing. A new study from Wuhan University, for instance, estimates that the market for dubious science-publishing activities, such as ghostwriting papers on nonexistent research, was of the order of 1 billion renminbi (US\$150 million) in 2009 - five times the amount in 2007. In other studies, one in three researchers surveyed at major universities and research institutions admitted to committing plagiarism, falsification or fabrication of data.

"The extent of the misconduct is disturbing," says Nicholas Steneck, director of the Research Ethics and Integrity Program at the University of Michigan in Ann Arbor. "It highlights the challenges China faces as it struggles to rapidly improve the research capacity of a very large system - with significant variations in quality - to be a world-class player in science."

Two weeks ago, reacting to the retractions of the crystallography papers, Jinggangshang University fired the correspondent authors, Zhong Hua and Liu Tao. It is unclear whether their coauthors, who include researchers from other institutions in China, will also be investigated.

The journal's editors say that the discrepancies came to light during tests of software designed to flag possible errors and unusual chemical features, such as abnormal distances between atoms. The software identified a large number of crystal structures that didn't make sense chemically; further checking, the editors say, suggests that the authors simply changed one or more atoms of an existing compound of known structure, then presented that structure as new. Zhong and Liu could not be reached for comment.

Editors at the journal are now checking the authenticity of other published crystal structures, including all submissions from Jinggangshan University.

Half of the 200,000-odd crystal structures published by the journal during the past five years have come from China. William Harrison, a chemist at the University of Aberdeen,
UK, who is one of three section editors for the journal, would not discuss the ongoing investigation but says that the generation of large numbers of structures by one group would not necessarily raise questions, because diffractometers can easily collect a couple of data sets a day. "In terms of papers submitted to Acta E, the vast majority coming from China are correctly determined structures, and they make a valuable contribution to science," he says.

Nevertheless, the Wuhan University study suggests that misconduct could be widespread in many fields. The team, led by computer scientist Shen Yang, used website analyses and onsite investigations to identify a wide range of dubious publishing activities. These include ghostwriting theses and academic papers on fictional research, bypassing peer-review for payment, and forging copies of legitimate Chinese or international journals.

The researchers analysed the most popular 800 websites involved in such activities - which together rack up 210,000 hits a day — and found that the cost of each transaction is typically 600 12,000 renminbi. Three-quarters of the demand comes from universities and institutions, says Shen. "There is a massive production chain for the entire publishing process," he says.

Concerned by such trends, China's science ministry commissioned a survey of researchers,

\section{Streamlined chemical tests rebuffed}

Europe's chemical regulator is threatening to stall safety studies that toxicologists say could prevent millions of animals being used in tests over the next eight years.

European Union (EU) legislation requires that chemicals be tested in two generations of animals to assess the effects on their reproductive systems. But proposed new tests would allow just one generation of animals to be used, with additional tests on a second generation required only if the first round raised concerns.

But the European Chemicals Agency (ECHA) in Helsinki, which implements the EU's REACH (Registration, Evaluation,
Authorisation and Restriction of Chemicals) legislation and is responsible for issuing guidance on tests, says that there is not yet enough evidence to rely on one-generation testing.

Toxicologists who advocate the switch, however, say that their scientific case is "overwhelming". They argue that REACH will fail unless the new test is adopted, because the two-generation tests are too expensive, demand too many animals, and require laboratory space and manpower that the EU simply doesn't have.

"Everyone is longing for this change to happen. It's the only way to make the legislation feasible," says toxicologist Thomas Hartung, former head of the European Centre for the Validation of Alternative Methods in Ispra, Italy, and now at Johns Hopkins Bloomberg School

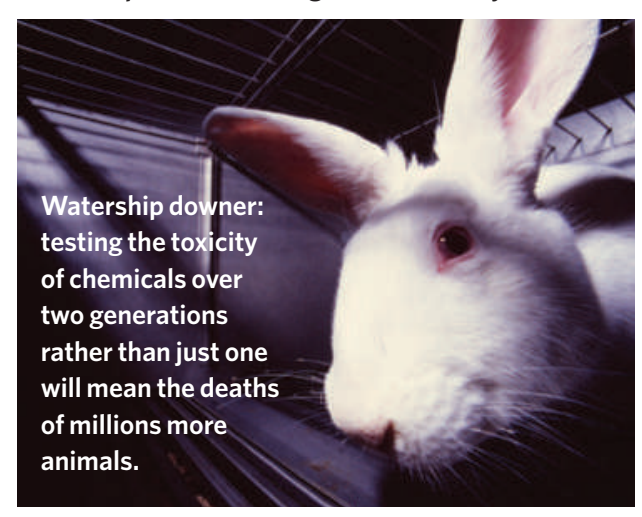

of Public Health in Baltimore, Maryland. He estimates that using the extended one-generation study would reduce animal use in REACH by $40-60 \%$. "Costs would be reduced in a similar range," he says.

"We can't perform all the two-generation studies that we are required to perform under REACH," adds Susanne Böhn, a toxicologist for BASF, a chemical company headquartered in 
